\title{
CDC Grand Rounds: A Public Health Approach to Detect and Control Hypertension
}

\author{
Rikita Merai, MPH ${ }^{1,2}$; Claudia Siegel, MPA ${ }^{3}$; Michael Rakotz, MD ${ }^{4}$; Peter Basch, MD ${ }^{5}$; Janet Wright, MD ${ }^{1}$; Betty Wong, DHSc ${ }^{6}$; Phoebe Thorpe, MD ${ }^{6}$
}

Hypertension is generally defined as systolic blood pressure $\geq 140 \mathrm{mmHg}$, or diastolic blood pressure $\geq 90 \mathrm{mmHg}$. A person who currently uses blood pressure-lowering medication is also defined as having hypertension. Hypertension is a leading risk factor for cardiovascular disease and stroke $(1,2)$. Hypertension affects nearly one third of U.S. residents aged $\geq 18$ years (approximately 75 million persons), and in approximately one half of adults with hypertension (nearly 35 million persons), it is uncontrolled (2). Among these 35 million U.S. residents with uncontrolled hypertension, 33\% (11.5 million persons) are not aware of their hypertension, 20\% (7 million persons) are aware of their hypertension, but are not being treated for it, and approximately 47\% (16.1 million persons) are aware of their hypertension and being treated for it, but treatment (by medication and/or lifestyle modification) is not adequately controlling their blood pressure (Figure) (2).

Uncontrolled hypertension is associated with increased cardiovascular morbidity and mortality and an increased use of health care resources (3), with approximately $\$ 49$ billion spent annually in direct and indirect medical expenses (4). Seventy-seven percent of persons who have a first stroke have hypertension (4). Of the 35 million U.S. residents who have uncontrolled hypertension, $81 \%$ (30 million) have health insurance, $83 \%$ (31 million) have a usual source of care, and $79 \%$ (30 million) received medical care in the previous year (Table). These data point to gaps and barriers in the current system that, if addressed, could lead to improved control of hypertension in the U.S. population, and considerable reduction in hypertension-associated morbidity and mortality.

Accurate diagnosis and appropriate management of hypertension require multiple reliable blood pressure readings over time. Missed opportunities to detect and control hypertension can arise at the individual level, the care provider level, and at the health care system level. Persons might not recognize that hypertension is uncontrolled because they do not experience symptoms. Many persons find it challenging to follow proper medication regimens, or make appropriate lifestyle changes such as eating a healthy, low-sodium diet and quitting smoking (5). During office visits, providers often must address other pressing issues, and might miss opportunities to reinforce the importance of, and strategies for, effective blood pressure control. At the health care system level, lack of access to blood pressure readings done elsewhere within a health care system, or concern over the reliability of those readings can lead to missed opportunities to diagnose and treat hypertension (G). These varied reasons for lack of hypertension control underscore the need for multiple approaches and a more coordinated effort to reduce the prevalence of hypertension and its associated morbidity and mortality.

The Million Hearts Initiative (Million Hearts) is a U.S. Department of Health and Human Services initiative, that focuses on efforts of federal agencies, state and local governments, health care providers and systems, community-based organizations, employers, and persons, with the overall goal of preventing one million heart attacks and strokes by 2017 (7). Hypertension is a major modifiable risk factor for heart attacks and strokes; thus, one major Million Hearts objective is to increase by 10 million the number of persons in the United States whose hypertension is under control (7). To achieve this, Million Hearts aims to enhance detection and control of hypertension by facilitating more accurate blood pressure measurement and monitoring, improving blood pressure treatment, and increasing awareness of hypertension in populations considered at increased risk. This report summarizes specific efforts to achieve these aims in two health care systems and a public health department.

NorthShore University Health System. NorthShore University Health System, in Illinois, is a health care delivery system that includes $>800$ physicians, four community hospitals, and a research institute; NorthShore uses an integrated and unified electronic health record (EHR) that stores data and makes data accessible across the entire health system. The NorthShore Undiagnosed Hypertension Quality Improvement Project was established to improve screening to identify patients with elevated blood pressure; ensure accuracy and reliability of office blood pressure measurement; recognize patients at risk at the point of care; and provide clinical decision support tools to facilitate appropriate treatment. NorthShore's Ambulatory Primary Care Innovations Group and clinical informatics team developed an enterprise-wide hypertension surveillance system within the EHR, which included point-of-care alerts for both clinic staff and physicians. Data were queried from the EHR using several unique algorithms to identify patients meeting the criteria for elevated blood pressure, and who might have hypertension (8). These algorithms, called NorthShore hypertension criteria, were derived from accepted clinical practices, guidelines, and research literature. Patients satisfying at least one of the hypertension criteria were notified that 
FIGURE. Number and percentage of adults with hypertension and uncontrolled hypertension, by awareness and treatment — National Health and Nutrition Examination Survey, United States, 2013-2014

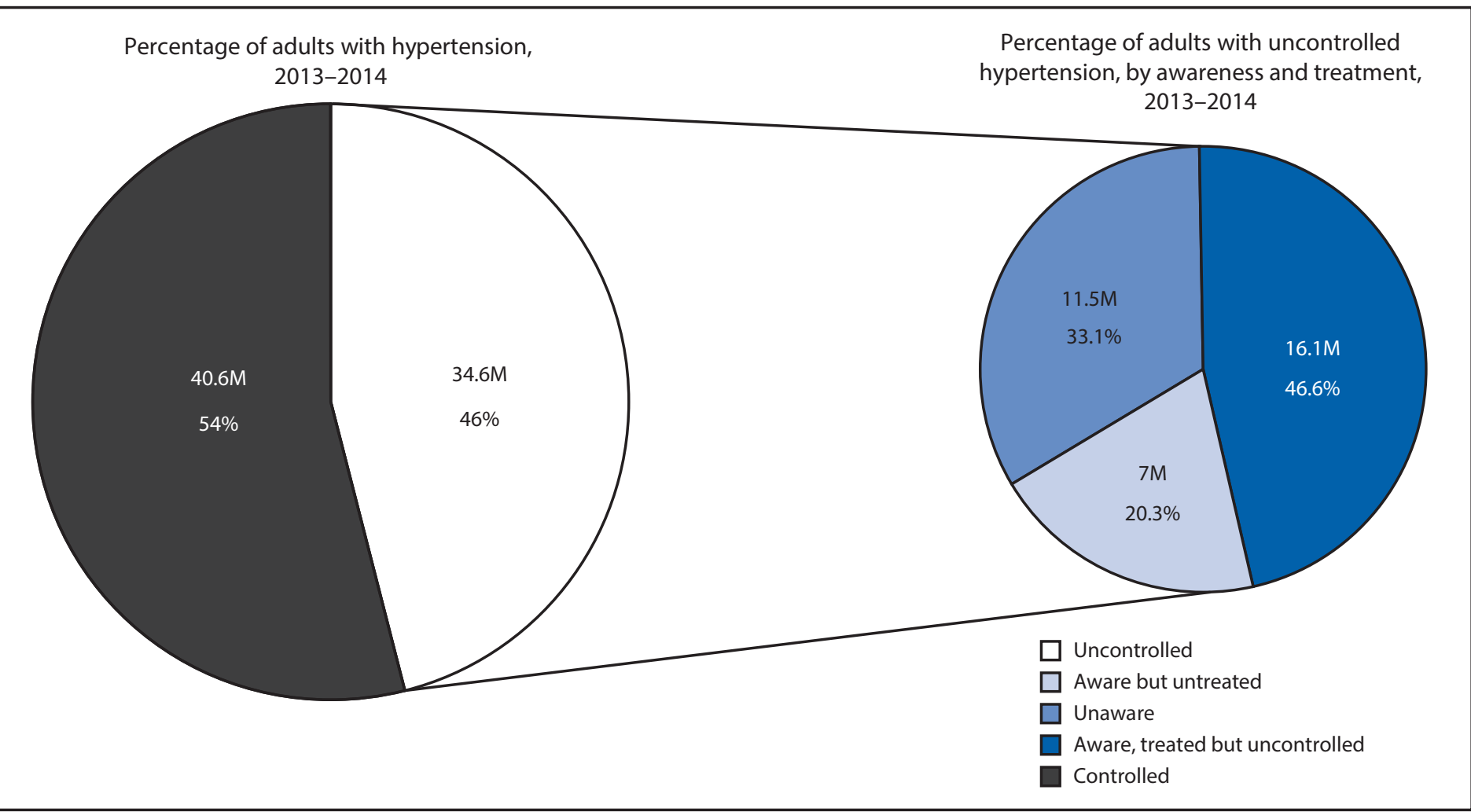

Abbreviation: $\mathrm{M}=$ million.

they might be at risk for hypertension, and urged to schedule an automated office blood pressure (AOBP) visit. At AOBP visits, a consistent protocol for measuring blood pressure was followed to verify whether patients had hypertension. Among patients who satisfied the NorthShore hypertension criteria, but chose not to come in for an AOBP visit, electronic "best practice advisories" alerted clinic staff and physicians each time the patient came for an office visit that the patient remained at risk for hypertension. These alerts continued until either an AOBP was performed, or a blood pressure-related diagnosis was placed in the EHR.

During the first 8 months of the project, 435 (52\%) of 836 patients with previously undiagnosed and untreated hypertension received a hypertension diagnosis using the algorithm alerts and confirmatory AOBP readings across the system's 23 primary care clinics. For patients meeting the NorthShore hypertension criteria, triggering recall to the office, $97 \%$ now have a diagnosis of hypertension in their EHR, and 94\% of persons with newly diagnosed hypertension were prescribed medication within 90 days of diagnosis. Screening for patients with undiagnosed hypertension using EHR data, combined with electronic alerts at the point of care was effective in this health care setting and reduced the number of undiagnosed hypertensive patients.

MedStar Health. MedStar Health is the largest healthcare system in the Maryland and Washington, D.C. area with 10 hospitals, ambulatory care and urgent care centers, approximately 100 community locations, and during 2012, >160,000 hospital admissions and 1.5 million outpatient visits. MedStar typically records a patient's blood pressure at every visit. To improve hypertension control, MedStar implemented a systemwide EHR prompt with suggested guidelines recommending default blood pressure goals, which took into account comorbid conditions, (e.g., diabetes, prior stroke, or acute myocardial infarction). If the provider did not further personalize the recommended blood pressure goal, the default blood pressure goal would autoinsert into the EHR. Once a guideline recommended blood pressure goal was inserted into a patient's record, every subsequent visit to any primary care doctor within the MedStar system presented an opportunity to verify that the patient's blood pressure was at the specified goal. Through the use of additional prompts, alerts, and patient handouts that were automatically generated, providers and patients were made aware that a patient's blood pressure did or did not meet the 
TABLE. Access to health care among 35 million persons with uncontrolled hypertension - National Health and Nutrition Examination Survey, United States, 2011-2014

\begin{tabular}{lc}
\hline Characteristic & No. (millions) \\
\hline Have health insurance & \\
Yes & 29.9 \\
No & 4.7 \\
Have usual source of health care & 30.8 \\
Yes & 3.9 \\
No & \\
No. of health care visits during past year & 25.3 \\
$\geq 2$ & 4.7 \\
1 & 4.7 \\
\hline
\end{tabular}

Source: CDC. National Health and Nutrition Examination Survey Data 2011-2014 (http://www.cdc.gov/nchs/nhanes.htm).

recommended goal, and patients were informed if additional consultations or treatments should be considered.

One year into MedStar's program to improve hypertension control, the percentage of patients with hypertension and a documented evidence-based blood pressure goal increased from $5 \%$ to $92 \%$. The percentage of patients with uncontrolled hypertension declined during this same period, from $32 \%$ to $28 \%$. Although these preliminary results represent a modest improvement in hypertension control, MedStar has begun evaluating best practices from high-performing providers and assessing the characteristics of patients with uncontrolled hypertension to identify potential next steps to further reduce uncontrolled hypertension.

Philadelphia Department of Public Health. Philadelphia has one of the highest hypertension prevalence rates in the nation, (44.7\% of adult males and $47.6 \%$ of adult females have hypertension) and has one of the highest rates of uncontrolled hypertension (18.9\% of adult males and $21.4 \%$ of adult females) in the United States (9).

In 2010, the Philadelphia Department of Public Health $(\mathrm{PDPH})$, through a CDC cooperative agreement, sought to improve public health data infrastructure to gather, store, analyze, and share more and higher quality data for the entire Philadelphia jurisdiction; the agreement focused on hypertension, adult immunization, and cancer screening. The Office of Health Information and Improvement was established within the Health Commissioner's Office, and the PDPH assembled a work group to secure hypertension data. The workgroup included state agencies, academic health systems, the prison system, the county medical society, the federation representing a majority of the Federally Qualified Health Centers, a community health service not-for-profit organization, and the regional hospital association. The Office of Health Information and Improvement asked this work group to share deidentified, aggregated data for their total patient population. The requested data elements included age, sex, race/ethnicity, and insurance type (if known); the number of persons within the patient population aged $\geq 18$ years who could be identified as hypertensive (using International Classification of Diseases, 9th Revision, Clinical Modification [ICD-9-CM] codes); and the number of patients who had their blood pressure under control $(\leq 140 / 90 \mathrm{mmHg})$. In response, partners provided prevalence data from EHRs, or administrative databases, along with existing reports, such as the Uniform Data System.

Data were also received from insurers covering 585,922 of Philadelphia's population aged $\geq 18$ years (just under $50 \%$ of that age cohort). Overall, hypertension prevalence was $24.2 \%$, which was lower than national survey data; however, this might be related to the age distribution of the insured population; $56.1 \%$ of the insured patients were aged $<44$ years. The percentage of the hypertensive population with adequately controlled hypertension among payer groups ranged from $35.4 \%$ to $62.5 \%$, possibly because of the variety of proxy measures used (e.g., medication adherence data). Among the payer groups, the population with Medicare had the highest prevalence of hypertension $(79.5 \%)$ and the highest prevalence of hypertension control (62.5\%). Provider data covered 355,057 adults aged $\geq 18$ years, with an average hypertension prevalence of $17.1 \%$ and control ranging from $42.1 \%$ to $65.7 \%$.

This data exchange enabled PDPH to have a better understanding of the variations in hypertension prevalence and control, identified considerable gaps in control, and provided an impetus to improve. By looking beyond the realm of traditional public health, PDPH and its partners are working to expand dialogue, data exchange, and collaborations to improve overall system performance in detecting and controlling high blood pressure in this high-need population.

Reaching the Million Hearts hypertension goal of 10 million more persons in the United States whose hypertension is under control will require more of these innovative efforts in health systems and communities. Collaborative and coordinated efforts are required to leverage the strengths and resources of both public health and health care systems. The NorthShore and MedStar Health examples demonstrate how health information technology tools can be used to help busy health care providers and their patients more effectively detect and control high blood pressure. PDPH demonstrates how public health can engage stakeholders (e.g., insurers, healthcare systems) to work together across systems to address this problem.

\footnotetext{
${ }^{1}$ Division for Heart Disease and Stroke Prevention, National Center for Chronic Disease Prevention and Health Promotion, CDC; ${ }^{2}$ IHRC, Inc. Atlanta, Georgia; ${ }^{3}$ Philadelphia Department of Public Health; ${ }^{4}$ Northwestern Memorial Physicians Group, Chicago, Illinois; ${ }^{5} \mathrm{MedStar}$ Health, Columbia, Maryland; ${ }^{6}$ Office of the Associate Director for Science, CDC.

Corresponding author: Janet Wright, JWright2@cdc.gov, 202-834-7901.
} 


\section{Acknowledgment}

Cathleen Gillespie, Division for Heart Disease and Stroke Prevention, National Center for Chronic Disease Prevention and Health Promotion, CDC.

\section{References}

1. Roger VL, Go AS, Lloyd-Jones DM, et al.; American Heart Association Statistics Committee and Stroke Statistics Subcommittee. Heart disease and stroke statistics-2012 update: a report from the American Heart Association. Circulation 2012;125:e2-220. http://dx.doi.org/10.1161/ CIR.0b013e31823ac046

2. Yoon SS, Fryar C, Carrol M. Hypertension prevalence and control among adults: United States, 2011-2014. National Center for Health Statistics data brief, November 2015;(220):1-8.

3. Chobanian AV, Bakris GL, Black HR, et al.; National High Blood Pressure Education Program Coordinating Committee. Seventh report of the Joint National Committee on Prevention, Detection, Evaluation, and Treatment of High Blood Pressure. Hypertension 2003;42:1206-52. http://dx.doi. org/10.1161/01.HYP.0000107251.49515.c2
4. Mozaffarian D, Benjamin EJ, Go AS, et al.; Writing Group Members; American Heart Association Statistics Committee; Stroke Statistics Subcommittee. Heart disease and stroke statistics-2016 update: a report from the American Heart Association. Circulation 2016;133:e38-360. http://dx.doi.org/10.1161/CIR.0000000000000350

5. CDC. 2010 Surgeon General's Report—how tobacco smoke causes disease: the biology and behavioral basis for smoking-attributable disease. Atlanta, GA: U.S. Department of Health and Human Services, CDC; 2016.

6. Wofford MR, Minor DS. Hypertension: issues in control and resistance. Curr Hypertens Rep 2009;11:323-8. http://dx.doi.org/10.1007/ s11906-009-0055-0

7. Frieden TR, Berwick DM. The "Million Hearts" initiative-preventing heart attacks and strokes. N Engl J Med 2011;365:e27. http://dx.doi. org/10.1056/NEJMp1110421

8. Rakotz MK, Ewigman BG, Sarav M, et al. A technology-based quality innovation to identify undiagnosed hypertension among active primary care patients. Ann Fam Med 2014;12:352-8. http://dx.doi.org/10.1370/afm. 1665

9. Olives C, Myerson R, Mokdad A, Murray C, Lim S. Prevalence, awareness, treatment and control of hypertension in United States counties, 20012009. PLoS One 2013;8(4)e60308. Epub April 5, 2013. http://dx.doi. org/10.1371/journal.pone.0060308 